\title{
A Prospective Evaluation of Two Defibrillation Safety Margin Techniques in Patients with Low Defibrillation Energy Requirements
}

S. ADAM STRICKBERGER, M.D., K. CHING MAN, D.O., JOSEPH SOUZA, M.D., ADAM ZIVIN, M.D., RAUL WEISS, M.D., BRADLEY P. KNIGHT, M.D., RAJIVA GOYAL, M.D., EMILE G. DAOUD, M.D., and FRED MORADY, M.D.

From the University of Michigan Medical Center, Ann Arbor, Michigan

Low-Energy Defibrillation. Introduction: In patients undergoing defibrillator implantation, an appropriate defibrillation safety margin has been considered to be either $10 \mathrm{~J}$ or an energy equal to the defibrillation energy requirement. However, a previous clinical report suggested that a larger safety margin may be required in patients with a low defibrillation energy requirement. Therefore, the purpose of this prospective study was to compare the defibrillation efficacy of the two safety margin techniques in patients with a low defibrillation energy requirement.

Methods and Results: Sixty patients who underwent implantation of a defibrillator and who had a low defibrillation energy requirement $(\leq 6 \mathrm{~J})$ underwent six separate inductions of ventricular fibrillation, at least 5 minutes apart. For each of the first three inductions of ventricular fibrillation, the first two shocks were equal to either the defibrillation energy requirement plus $10 \mathrm{~J}(14.6 \pm 1.0 \mathrm{~J})$, or to twice the defibrillation energy requirement $(9.9 \pm 2.3 \mathrm{~J})$. The alternate technique was used for the subsequent three inductions of ventricular fibrillation. For each induction of ventricular fibrillation, the first shock success rate was $99.5 \% \pm 4.3 \%$ for shocks using the defibrillation energy requirement plus $10 \mathrm{~J}$, compared to $95.0 \% \pm 17.2 \%$ for shocks at twice the defibrillation energy requirement $(P=0.02)$. The charge time $(P<0.0001)$ and the total duration of ventricular fibrillation $(P<0.0001)$ were each approximately 1 second longer with the defibrillation energy requirement plus $10 \mathrm{~J}$ technique.

Conclusion: This study is the first to compare prospectively the defibrillation efficacy of two defibrillation safety margins. In patients with a defibrillation energy requirement $\leq 6 \mathrm{~J}$, a higher rate of successful defibrillation is achieved with a safety margin of $10 \mathrm{~J}$ than with a safety margin equal to the defibrillation energy requirement. ( Cardiovasc Electrophysiol, Vol. 9, pp. 41-46, January 1998)

ventricular fibrillation, ventricular defibrillation, probability of defibrillation, implantable cardioverter defibrillator

\section{Introduction}

At the time of defibrillator implantation, a variety of techniques are used to assess defibrillation efficacy and to program defibrillation safety margins. ${ }^{1-4}$ Although implantable defibrillators can sim-

Dr. Strickberger is a paid consultant to Guidant, Inc.

Address for correspondence: S. Adam Strickberger, M.D., University of Michigan Medical Center, 1500 East Medical Center Dr., Ann Arbor, MI 48109-0022. Fax: 313-936-7026.

Manuscript received 3 October 1997; Accepted for publication 20 November 1997. ply be programmed to deliver the maximum available energy, there are advantages to using the lowest energy consistently associated with successful defibrillation. In current clinical practice, an appropriate defibrillation safety margin in patients undergoing defibrillator implantation has been defined either as $10 \mathrm{~J}$ or as an energy equal to the defibrillation energy requirement. The results of a previous clinical study suggested that a larger safety margin may be required when the defibrillation energy requirement is $\leq 6 \mathrm{~J}$ than when it is $>6$ $\mathrm{J} .^{5}$ The purpose of this prospective study was to compare the defibrillation efficacy of a safety mar- 
gin of $10 \mathrm{~J}$ with that of a safety margin equal to the defibrillation energy requirement in patients with a low defibrillation energy requirement.

\section{Methods}

\section{Patient Population}

The study population consisted of 60 patients undergoing evaluation of a defibrillation energy requirement. The defibrillation energy requirement was determined prior to hospital discharge after implantation of an implantable defibrillator in 32 patients, 2 months after implantation of an implantable defibrillator in 15 patients, and 1 year after a defibrillator was implanted in 13 patients. The mean age of the patients was $62 \pm 13$ years, and 46 were men. Thirty-three patients had coronary artery disease, 20 had idiopathic cardiomyopathy, 3 had other forms of cardiomyopathy, and 4 had no structural heart disease. The mean left ventricular ejection fraction was $0.29 \pm 0.13$. The presenting symptom was cardiac arrest or syncope in 32 patients and ventricular tachycardia in 28 patients. Eight patients were being treated with amiodarone, and no patient was being treated with a Class I antiarrhythmic agent.

\section{Defibrillator System}

The patients provided informed consent under a protocol approved by the Human Research Committee at the University of Michigan. All patients came to the operating room in a postabsorptive state.

An endocardial defibrillation lead with a distal electrode of $295 \mathrm{~mm}^{2}$ and a proximal electrode of $617 \mathrm{~mm}^{2}$, separated by a distance of $11.5 \mathrm{~cm}$, was used in this study (Endotak model 125, Cardiac Pacemakers, Inc., St. Paul, MN, USA). Under fluoroscopic guidance, the defibrillation lead was positioned in the right ventricular apex via a subclavian or cephalic vein. The distal shocking coil was placed in the right ventricular apex, and the proximal shocking coil was positioned in the right atrium or at the junction of the right atrium and the superior vena cava.

Each patient in this study received a defibrillator with a truncated, fixed-tilt biphasic waveform with a first phase tilt of $60 \%$ and a second phase tilt of $50 \%$. The generator functioned as a shocking electrode in 46 patients. Only one patient had a subcutaneous shocking electrode, and in this patient the generator did not function as a defibrillation electrode.
During the implantation procedure, a step-down defibrillation protocol was utilized to determine the defibrillation energy requirement. The defibrillation energy requirement was defined as the lowest energy that successfully converted ventricular fibrillation to sinus rhythm. A defibrillation energy requirement considered adequate for device implantation was at least $10 \mathrm{~J}$ less than the maximum output of the defibrillator. Shock energies of $20,15,10,5,3$, and $1.0 \mathrm{~J}$ were delivered until ventricular fibrillation failed to convert to sinus rhythm. The shocks were delivered directly from the implantable defibrillator. Ventricular fibrillation was induced by ventricular pacing with a $15-\mathrm{V}$ pulse with a duration of $1.1 \mathrm{msec}$ delivered every $30 \mathrm{msec}$ for 1 to 3 seconds. Shocks were delivered after ventricular fibrillation was sensed by the device. At least 5 minutes were allowed to elapse between each induction of ventricular fibrillation.

\section{Study Protocol}

At the time of the study protocol, the patients were brought to the electrophysiology laboratory in a postabsorptive state. All inductions and conversions of ventricular fibrillation were performed using the implanted defibrillator. At least 5 minutes were allowed to elapse between each induction of ventricular fibrillation. The delivered energies were $20,15,10,6,5,4,3,2$, and $1 \mathrm{~J}$. The shock energy selected for the first defibrillation was the energy equal to the defibrillation energy requirement at the time of device implantation. The step-down defibrillation energy requirement was then determined using the decrements described above. The defibrillation energy requirement was defined as the lowest energy successful at converting ventricular fibrillation to sinus rhythm. If the defibrillation energy requirement was $6 \mathrm{~J}$ or less, the study protocol was performed. Twentysix percent of the patients who underwent a defibrillator evaluation with a determination of the defibrillation energy requirement had a defibrillation energy requirement that allowed participation in the study protocol.

The study protocol required six inductions of ventricular fibrillation. The patients were randomly assigned to 1 of 2 safety margins for the first three inductions of ventricular fibrillation. The alternate technique was used for the subsequent three ventricular fibrillation inductions. One technique was designed to test the efficacy of a 10-J safety margin, and the shock energy was determined by 
adding $10 \mathrm{~J}$ to the defibrillation energy requirement. A $10-\mathrm{J}$ safety margin in patients with low defibrillation energy requirements will be associated with shock energies that are at least 2.5 times the defibrillation energy requirement. A safety margin equal to the defibrillation energy requirement was the second technique. The shock energy in this technique was determined by doubling the defibrillation energy requirement. The programmed shock energy for each of the first two shocks for each induction of ventricular fibrillation was either the defibrillation energy requirement plus $10 \mathrm{~J}$, or the energy equal to twice the defibrillation energy requirement. At least 5 minutes were allowed to elapse between each induction of ventricular fibrillation. The success of each shock was noted. The total duration of ventricular fibrillation, the defibrillator charge time, and the shocking impedance were recorded for each induction of ventricular fibrillation.

\section{Statistical Analysis}

Continuous variables are expressed as mean \pm $1 \mathrm{SD}$ and were compared using a paired or unpaired $t$-test, as appropriate. Multiple continuous variables were compared by ANOVA; and nominal variables were compared by Chi-square analysis. A probability value less than 0.05 was considered statistically significant.

\section{Results}

\section{First Shock Defibrillation Efficacy}

The mean defibrillation energy requirement was $5.0 \pm 1.1 \mathrm{~J}$ in the entire study population of $60 \mathrm{pa}-$ tients. The programmed energy for each of the first two shocks was $14.6 \pm 1.0 \mathrm{~J}$ in the $10-\mathrm{J}$ safety margin group. This was equivalent to a multiple of $3.1 \pm 0.8$ times the defibrillation energy requirement (range 2.5 to 6.0 ). When using a safety margin equal to the defibrillation energy requirement, the mean tested energy was $9.9 \pm$ $2.3 \mathrm{~J}$.

The overall first shock success rate with the defibrillator programmed to the defibrillation energy requirement plus $10 \mathrm{~J}$ was $99.5 \% \pm 4.3 \%$, compared to $95.0 \% \pm 17.2 \%$ for a first shock programmed to twice the defibrillation energy requirement $(P=0.02)$. There was only one failed first shock defibrillation attempt with a safety margin of $10 \mathrm{~J}$, whereas nine failed attempts were noted in six patients in whom the safety margin was equal to the defibrillation energy requirement (Table 1).

\section{First and Second Shock Defibrillation Efficacy}

The probability that an episode of ventricular fibrillation would be successfully defibrillated with either the first or the second shock was not significantly different between the defibrillation energy requirement plus $10 \mathrm{~J}(100 \% \pm 0 \%)$ and the defibrillation energy requirement $\times 2(98.8 \% \pm$ $6.0 \% ; \mathrm{P}=0.2)$ groups. There were two patients in whom the second shock with a safety margin of twice the defibrillation energy requirement was not effective (Table 1).

\section{Failed Defibrillation Attempts}

The defibrillation energy requirement in patients who had at least one failed defibrillation attempt was $4.5 \pm 1.9 \mathrm{~J}$, and was $5.0 \pm 1.2 \mathrm{~J}$ in patients with $100 \%$ successful defibrillation $(\mathrm{P}=$ 0.3 ). The likelihood of a failed shock did not cor-

\begin{tabular}{|c|c|c|c|c|c|}
\hline \multirow[b]{3}{*}{ Pt. No. } & \multirow[b]{3}{*}{$\operatorname{DER}(\mathbf{J})$} & \multicolumn{2}{|c|}{ TABLE 1} & & \\
\hline & & \multicolumn{2}{|c|}{$\begin{array}{c}\text { Efficacy of DER } \\
+10 \mathrm{~J} \text { Success }\end{array}$} & \multicolumn{2}{|c|}{$\begin{array}{l}\text { Efficacy of DER } \\
\times 2 \text { Success }\end{array}$} \\
\hline & & First Shock & Second Shock & First Shock & Second Shock \\
\hline 1 & 5 & $3 / 3$ & - & $2 / 3$ & $1 / 1^{*}$ \\
\hline 2 & 2 & $3 / 3$ & - & $2 / 3$ & $1 / 1$ \\
\hline 3 & 5 & $3 / 3$ & - & $2 / 3$ & $1 / 1$ \\
\hline 4 & 4 & $3 / 3$ & - & $2 / 3$ & $0 / 1$ \\
\hline 5 & 6 & $2 / 3$ & $1 / 1$ & $0 / 3$ & $3 / 3$ \\
\hline 6 & 5 & $3 / 3$ & - & $1 / 3$ & $1 / 2$ \\
\hline
\end{tabular}

$\mathrm{DER}=$ defibrillation energy requirement; DER + $10 \mathrm{~J}$ Success = successful defibrillations with defibrillation energy requirement plus $10 \mathrm{~J}$ shocks; DER $\times 2$ Success $=$ successful defibrillations with an energy of twice the defibrillation energy requirement.

* Second shock successful, but shock energy inadvertently programmed to $27 \mathrm{~J}$ instead of $10 \mathrm{~J}$.

Note: In each instance, the numerator represents the number of successful defibrillations, and the denominator represents the number of defibrillation attempts. 
relate with age $(P=0.3)$, gender $(P=0.3)$, ejection fraction $(P=0.9)$, type of structural heart disease $(P=0.7)$, presentation with cardiac arrest $(P=0.3)$, concurrent therapy with amiodarone ( $P$ $=0.6$ ), the use of a defibrillator can as a shocking electrode ( $P=0.1$ ), or the defibrillation energy requirement determined during defibrillator implantation $(\mathrm{P}=0.3)$.

\section{Charge Times and Duration of Ventricular Fibrillation}

The charge time $(4.2 \pm 0.5 \mathrm{sec})$ and the total duration of ventricular fibrillation $(8.6 \pm 1.0 \mathrm{sec})$ associated with a 10-J safety margin were significantly longer than the charge time $(2.9 \pm 0.8$ $\sec , \mathrm{P}<0.0001)$ and the total duration of ventricular fibrillation $(7.7 \pm 1.7 \mathrm{sec}, \mathrm{P}=0.0002)$ associated with a safety margin equal to the defibrillation energy requirement. The total duration of ventricular fibrillation before a successful first shock was $8.6 \pm 1.0$ seconds when the $10-\mathrm{J}$ safety margin technique was utilized, compared to $7.3 \pm$ 1.0 seconds $(\mathrm{P}<0.0001)$ when a safety margin equal to the defibrillation energy requirement was used. The shock impedance was similar for each of the two groups $(42.2 \pm 8.2 \Omega$ vs $42.5 \pm$ $8.3 \Omega, P=0.2$ ).

\section{Discussion}

\section{Major Findings}

This study is the first to prospectively compare the efficacy of two defibrillation safety margins in patients with a defibrillation energy requirement $\leq 6 \mathrm{~J}$. The results demonstrate that a higher rate of successful defibrillation is achieved with a safety margin of $10 \mathrm{~J}$ than with a safety margin equal to the defibrillation energy requirement. The higher probability of successful defibrillation with a 10-J safety margin is associated with an approximately 1 -second longer duration of ventricular fibrillation. If the defibrillation energy had been the maximum available energy, i.e., 27 to 29 $J$, then the charge time and the total duration of ventricular fibrillation would have been 3 to 4 seconds longer. Currently, approximately $25 \%$ of patients have defibrillation energy requirements $\leq$ $6 \mathrm{~J}$. Hence, these observations are of clinical importance because an objective approach to programming safety margins in patients with a defibrillation energy requirement $\leq 6 \mathrm{~J}$ is critical as advances in defibrillation result in improved lowenergy defibrillation efficacy.

\section{Probability of Successful Defibrillation}

Theoretically, uniformly successful defibrillation should occur with shock energies high on the slowly ascending portion of the probability of successful defibrillation curve. ${ }^{6-10}$ This part of the defibrillation energy requirement curve is commonly referred to as the "plateau." The term plateau, however, is a misnomer because the probability of successful defibrillation does not achieve a constant value. ${ }^{6-10}$ The results of animal and clinical studies suggest that the slowly ascending portion of the defibrillation energy requirement curve begins at a multiple of approximately 1.3 times the defibrillation energy requirement. ${ }^{5-10}$ In most patients, close to $100 \%$ successful defibrillation is expected with a shock energy of twice the defibrillation energy requirement. ${ }^{5}$ However, a shock energy greater than twice the defibrillation energy requirement may be required to achieve uniformly successful defibrillation in patients with a low defibrillation energy requirement. ${ }^{5}$ In the present study, a 10-J safety margin in patients with a low defibrillation energy requirement was associated with shock energies of 2.5 to 6.0 times the defibrillation energy requirement. The use of shock energies equal to larger multiples of the defibrillation energy requirement likely explains the increased probability of successful defibrillation observed with the 10-J safety margin. In patients with higher defibrillation energy requirements, the probability of successful defibrillation with a 10-J safety margin will likely decrease according to the multiple of the defibrillation energy requirement. 5.9 .10

\section{Previous Studies}

A number of animal studies and a single human study have addressed the issue of defibrillation energy requirement curves and safety margins..$^{5-10}$ In humans, a step-down defibrillation energy requirement identifies the energy associated with $70 \%$ probability of successful defibrillation. ${ }^{5}$ The results of previous experimental and clinical trials suggest that essentially uniformly successful defibrillation is achieved with an energy of twice the defibrillation energy requirement, although the defibrillation energy requirement curve for an animal or a patient with a low defibrillation energy requirement is narrower and has a greater slope than for an animal or a patient with a higher defibrillation energy requirement.5,9,10 Therefore, doubling a low defibrillation energy requirement that is slightly underestimated could result in a significantly reduced probability of successful defi- 
brillation at that shock energy. This is probably the explanation for why patients with low defibrillation energy requirements occasionally require a larger defibrillation safety margin than patients with higher defibrillation energy requirements.

The data that support the use of a 10-J safety margin are limited. In patients at high risk for lethal ventricular arrhythmias, implantable defibrillator therapy has been shown to reduce the risk of sudden cardiac death if an empiric safety margin of $10 \mathrm{~J}$ is achieved. ${ }^{11-15}$ Neither clinical nor experimental studies have directly addressed the issue of absolute energy margins; however, the probability of successful defibrillation can be estimated based on a multiple of the defibrillation energy requirement. Assuming a defibrillation energy requirement $\leq 25 \mathrm{~J}$, the conventional 10-J safety margin provides a safety margin energy equal to 0.4 times the defibrillation energy requirement with a 35-J output defibrillator, and in humans is associated with a high probability of successful defibrillation. ${ }^{5}$ The risk of sudden death and total mortality is higher in patients in whom a 10-J safety margin, or a shock energy less than 1.2 times the defibrillation energy requirement, cannot be achieved. ${ }^{16,17}$ This implies that shock energies of a multiple greater than this are required for appropriate clinical efficacy. ${ }^{16,17}$

\section{Limitations}

A limitation of this study is that the data were obtained using the same endocardial lead and biphasic waveform. Therefore, these data may not be applicable to defibrillation using other lead systems and waveforms.

\section{Clinical Implications}

A rational approach to defibrillator programming in patients with a low defibrillation energy requirement is to program the first shock energy equal to the defibrillation energy requirement plus $10 \mathrm{~J}$. Confirmation of defibrillation efficacy is probably not required with a $10-\mathrm{J}$ safety margin because of the probability of nearly universally successful defibrillation observed with this safety margin technique in patients with low defibrillation energy requirements. Alternatively, one could program the device to an energy equal to twice the defibrillation energy requirement; however, with this lower safety margin technique it may be important to confirm defibrillation efficacy. Programming the shock energy to the maximum available energy irrespec- tive of the defibrillation energy requirement results in several additional seconds of ventricular fibrillation and is probably not necessary. Using the lowest effective defibrillation safety margin will allow therapy to be delivered more quickly, may prevent syncope, may prolong device longevity, and may be hemodynamically beneficial for patients with a biphasic defibrillator. ${ }^{18-23}$

Acknowledgments: The authors would like to thank Allyson Navyac for her secretarial support, and Seema Sonnad, Ph.D., from the Consortium for Health Outcomes, Innovations and Cost Effectiveness Studies, at the University of Michigan Medical Center.

\section{References}

1. Rattes MF, Jones DL, Sharma AD, et al: Defibrillation threshold: A simple and quantitative estimate of the ability to defibrillate. PACE 1987;10:70-77.

2. Lehmann MH, Steinman RT, Schuger CD, et al: Defibrillation threshold testing and other practices related to AICD implantation: Do all roads lead to Rome? PACE 1989;12:1530-1537.

3. Jones DL, Irish WD, Klein GJ: Defibrillation efficacy: Comparison of defibrillation threshold versus dose-response curve determination. Circ Res 1991;69:45-51.

4. Singer I, Lang D: Defibrillation threshold: Clinical utility and therapeutic implications. PACE 1992;15: 932-949.

5. Strickberger SA, Daoud EG, Davidson T, et al: Probability of successful defibrillation at multiples of the defibrillation energy requirement in patients with an implantable defibrillator. Circulation 1997;96:1525-1531.

6. McDaniel WC, Schuder JC: The cardiac ventricular defibrillation threshold: Inherent limitations in its application and interpretation. Med Instrum 1987;21: 170-176.

7. McDaniel WC, Schuder JC: An up-down algorithm for estimation of the cardiac ventricular defibrillation threshold. Med Instrum 1988;22:286-292.

8. Davy JM, Fain ES, Dorian P, et al: The relationship between successful defibrillation and delivered energy in open-chest dogs: Reappraisal of the defibrillation threshold concept. Am Heart J 1987; 1 13:77-84.

9. Jones DL: The defibrillation threshold: A reliable method for rapid determination of defibrillation efficacy. In Estes NAM, Manolis AS, Wang PJ, eds. Implantable Cardioverter-Defibrillators. Marcel Dekker, Inc., New York, 1994, pp. 29-54.

10. Lang DJ, Ken Knight BH: Implant support devices. In Singer I, ed. Implantable Cardioverter Defibrillator. Futura Publishing Company, Inc., Armonk, NY, 1994, pp. 223-253.

11. Mirowski M, Reid P, Winkle R, et al: Mortality in patients with implanted automatic defibrillators. Ann Intern Med 1983;98:585-588.

12. Echt D, Armstrong K, Schmidt P, et al: Clinical experience, complications and survival in 70 patients with 
the automatic implantable cardioverter/defibrillator. Circulation 1985;71:289-296.

13. Winkle R, Mead R, Ruder M, et al: Long-term outcome with the automatic implantable cardioverter defibrillator. J Am Coll Cardiol 1989;13:1353-1361.

14. Nisam S, Mower M, Thomas A, et al: Patient survival comparison in three generations of automatic implantable cardioverter defibrillators: Review of 12 years, 25,000 patients. PACE 1993;16:174-178.

15. Moss AJ, Hall WJ, Cannom DS, et al: Improved survival with an implanted defibrillator in patients with coronary disease at high risk for ventricular arrhythmia. N Engl J Med 1996;335:1933-1940.

16. Pinski SL, Vanerio G, Castle LW, et al: Patients with a high defibrillation threshold: Clinical characteristics, management, and outcome. Am Heart J 1991;122:89-95.

17. Epstein AE, Ellenbogen KA, Kirk KA, et al: Clinical characteristics and outcome of patients with high defibrillation thresholds. Circulation 1992;86:1206-1216.

18. Antunes ML, Spotnitz HM, Livelli FD, et al: Effect of electrophysiological testing on ejection fraction during cardioverter/defibrillator implantation. Ann Thorac Surg 1988;45:315-318.

19. Hachenberg T, Hammel D, Mollhoff T, et al: Cardiopulmonary effects of internal cardioverter/defibrillator implantation. Acta Anaesthesiol Scand 1991;35: 626-630.

20. Keyl C, Tassani P, Kemkes B, et al: Hemodynamic changes due to intraoperative testing of the automatic implantable cardioverter defibrillator: Implications for anesthesia management. J Cardiothorac Vasc Anesth 1993;7:442-447.

21. Steinbeck G, Dorwarth U, Mattke S, et al: Hemodynamic deterioration during ICD implant: Predictors of high risk patients. Am Heart J 1994;127:1064-1067.

22. Meyer J, Mollhoff T, Seifert T, et al: Cardiac output is not affected during intraoperative testing of the automatic implantable cardioverter defibrillator. J Cardiovasc Electrophysiol 1996;7:211-216.

23. Tokano T, Souza J, Knight BP, et al: The effect of ventricular shocks on cardiac output. (Abstract) J Am Coll Cardiol, 1997;29:257. 
This document is a scanned copy of a printed document. No warranty is given about the accuracy of the copy. Users should refer to the original published version of the material. 